\section{Prevenção do câncer de colo do útero: um modelo teórico para analisar o acesso e a utilização do teste de Papanicolaou}

\section{Cervical cancer prevention: a theoretical framework to analyze Papanicolaou test access and use}

Adriana de Araujo Pinho 1

Ivan França-Junior 1

1 Departamento de Saúde Materno-Infantil. Faculdade de Saúde Pública. Universidade de São Paulo. Av. Dr. Arnaldo 715, $2^{\circ}$ andar. São Paulo, SP, Brasil. CEP 01.246-904

\begin{abstract}
Several studies have indicated that the prevailing high incidence and mortality rates of cervical cancer, mainly in developing countries, are due to the low quality and coverage of the cervical smear or Papanicolaou (Pap) test. This article intends to analise some aspects related to measures of control and prevention of cervical cancer, such as the effectiveness of the Pap test, the operational and scientific rationale of public health policies for the prevention of cervical cancer and coverage of the Papanicolaou test in many countries. It also intends to review the explanatory models that have been proposed to analyze access to this service. For this latter objective, we investigated the factors associated to the use of the Pap test described within the epidemiological literature and we proposed a new approach in the investigation of these factors, incorporating social, cultural and organizational aspects in the analysis of access to and use of this practice so that measures of prevention and control may be more coherent with the needs and the rights of women.
\end{abstract}

Key words Cervix neoplasms, Vaginal smears, Preventive health services, Health services
Resumo Vários estudos têm apontado que a permanência das altas taxas de incidência e mortalidade por câncer cérvico-uterino deve-se à baixa qualidade e cobertura do teste de Papanicolaou, principalmente em paises em desenvolvimento. Pretende-se neste artigo analizar alguns pontos relacionados às medidas de prevenção e controle do câncer cervical quanto à efetividade do teste de Papanicolaou, a lógica operacional e científica por detrás das políticas públicas de prevenção ao câncer cervical e a cobertura do teste em países norte-americanos, europeus e na América Latina. Consideram-se, ainda, os modelos explicativos que estão sendo propostos para avaliar o acesso e a utilização deste serviço, a partir da análise dos fatores associados à realização do teste de Papanicolaou descritos pela literatura. Propõe-se uma nova abordagem na investigação destes fatores, buscando a integração e interlocução de outros aspectos de cunho social, cultural e organizacional na análise do acesso e da utilização deste exame, visando um planejamento mais coerente das ações de prevenção e promoção à saúde com as necessidades e direitos das mulheres.

Palavras-chave Neoplasias do colo uterino, Esfrago vaginal, Serviços preventivos, Serviços de Saúde 
Há tempos, o câncer de colo do útero vem ocupando um lugar de destaque nas taxas de morbi-mortalidade entre a população feminina, especialmente nos países em desenvolvimento. No início da década de noventa, foram estimados 371.200 casos novos de câncer cervical invasivo no mundo, representando quase $10 \%$ de todos os cânceres entre a população feminina, sendo que $78 \%$ desses ocorreram em países em desenvolvimento, ${ }^{1}$ fazendo com que as taxas de incidência (ajustadas por idade) por esta doença nestes países permanecesse, desde 1985, em segundo lugar, perdendo somente para o câncer de mama, porém ocupando o primeiro lugar em países do sul e leste da África, da América Central, da região centro-sul da Ásia e na Melanésia. ${ }^{1}$

Particularmente na América Latina, as maiores taxas de incidência (ajustadas por idade) de câncer cervical, estimadas em 1990, foram observadas no Brasil, na cidade de Belém do Pará (64,8/100.000 mulheres), seguida pelo Peru, na cidade de Trujillo (53,5/100.000 mulheres). ${ }^{2}$ Quanto à taxa de mortalidade, segundo Eluf-Neto e Nascimento, ${ }^{3}$ não houve nenhuma tendência a redução significativa no período de 1960 a 1993 em países da América Latina e do Caribe, tais como Brasil, Colômbia, Cuba, Guatemala, Uruguai e Venezuela. No Brasil, particularmente no Estado de São Paulo, a taxa bruta de mortalidade por câncer de colo do útero se estabilizou em torno de 4,2/100.000 mulheres desde a década de 70,4 com um deslocamento de óbitos para as faixas etárias mais avançadas, acima de 70 anos. 5

As razões para a permanência de altas taxas de incidência e mortalidade por câncer de colo do útero em muitos países da América Latina e do Caribe encontram-se, provavelmente, no perfil epidemiológico que essa doença adquire nesses países, quanto à freqüência dos fatores de risco, mas, principalmente quanto ao grau de implementação de ações efetivas de curto e longo prazos tanto no plano técnico, no diagnóstico precoce da doença e tratamento das lesões detectadas, quanto nos planos educacional, social e político-econômico.

O planejamento das ações de intervenção e controle da doença se dá, prioritariamente, no plano técnico, pelo diagnóstico precoce das lesões precursoras através do teste de Papanicolaou, e se orientam pela distribuição dessas lesões segundo as faixas etárias das mulheres mais acometidas e pela periodicidade dos exames colpocitológicos, seguindo a lógica epidemiológica do risco e da relação custo-benefício/efetividade que norteiam as intervenções em saúde pública.

No entanto, o que se observa é que tais ações têm se mostrado limitadas e incoerentes com as reais ne- cessidades da população feminina de maior risco para a doença, a despeito do conhecimento de características epidemiológicas e fisiopatológicas do câncer cervical ter trazido possibilidades de intervenção técnica sobre a enfermidade e sua respectiva prevenção e controle, como o período assintomático da doença relativamente longo e passível de ser detectado precocemente. $\mathrm{O}$ acesso e a utilização do teste de Papanicolaou têm se confrontado com algumas barreiras presentes nos mais diferentes aspectos da vida das mulheres. Entender como se dá este processo tornou-se o objetivo principal deste estudo. Discute-se, inicialmente, de que modo uma ação de prevenção secundária - a realização do teste de $\mathrm{Pa}$ panicolaou - se formalizou como uma intervenção técnica legitimada cientificamente, a partir da análise de sua verdadeira efetividade em reduzir as taxas de incidência e mortalidade por câncer cervical e a lógica científica e operacional por detrás das políticas públicas de prevenção ao câncer cervical. Examina-se, ainda, a cobertura do teste de Papanicolaou descrita entre a população para a qual se destina, e quem, de fato, está sendo alcançado. Procurase observar ainda sob quais modelos explicativos têm sido investigados o acesso e a utilização deste exame, a partir da análise dos fatores associados à realização do teste descritos pela literatura epidemiológica. Propõe-se, por fim, um modelo contextual, que incorpore os modelos explicativos já existentes também como uma abordagem que integre outros elementos do plano social, cultural, organizacional e programático julgados essenciais no entendimento do acesso e utilização de serviços preventivos.

\section{O teste de Papanicolaou na prevenção e controle do câncer cervical}

As estratégias de prevenção secundária ao câncer de colo do útero consistem no diagnóstico precoce das lesões de colo uterino antes de se tornarem invasivas, a partir de técnicas de rastreamento ou screening compreendidas pela colpocitologia oncológica ou teste de Papanicolaou, colposcopia, cervicografia e, mais recentemente, os testes de detecção do DNA do vírus Papiloma humano em esfregaços citológicos ou espécimes histopatológicos. O exame colpocitológico ou teste de Papanicolaou, dentre os métodos de detecção, é considerado o mais efetivo e eficiente a ser aplicado coletivamente em programas de rastreamento do câncer cérvico-uterino, sendo uma técnica amplamente difundida há mais de 40 anos, mesmo sem ter sido objeto de avaliação por meio de estudos experimentais, como os ensaios clínicos 
aleatorizados.

A crença na efetividade do teste de Papanicolaou em reduzir as taxas de morbi-mortalidade por câncer cervical vem de duas fontes: de estudos comparativos de tendências temporais, mostrando a redução nas taxas de incidência e mortalidade por câncer cervical em diferentes países, seguida à introdução de programas populacionais de rastreamento do câncer cervical, especialmente em países escandinavos, 6 nos Estados Unidos e no Canadá, 7 e de estudos epidemiológicos do tipo caso-controle, indicando o risco de câncer cervical entre mulheres que nunca realizaram o teste de Papanicolaou e um aumento no risco de câncer proporcional ao tempo desde o último teste realizado. 8,9

Tais estudos ainda sugeriram que para que os programas de screening tivessem algum impacto na redução das taxas de incidência e mortalidade por câncer cérvico-uterino, haveria a necessidade de orientar as ações de intervenção pela lógica epidemiológica do risco aliada à análise custo-benefício, isto é, alcançando as mulheres com maior risco para o câncer cérvico-uterino, sem perder de vista uma distribuição otimizada dos recursos financeiros, especialmente naqueles países com escassez de recursos. Assim, o planejamento das ações de prevenção e controle da doença tem se orientado, prioritariamente, pela distribuição das lesões cervicais segundo as faixas etárias das mulheres mais acometidas pela doença, especialmente aquelas mais avançadas, entre 35 e 59 anos, e pela periodicidade do exame colpocitológico.

$\mathrm{O}$ argumento que permeia a priorização desta faixa etária nos programas de rastreamento do câncer cervical é baseado em estudos que mostram que rastrear mulheres muito jovens não teria impacto na redução da incidência por câncer cervical, visto que neste grupo populacional as lesões predominantes são de baixo grau e mais da metade regrediriam espontaneamente dentro de seis a dezoito meses. ${ }^{10}$ Como notou Day, 11 a partir de tendências temporais nas taxas de incidência por câncer cervical específicas por idade em diferentes países, o teste de Papanicolaou realizado entre 35 e 60 anos de idade tem se mostrado 30 vezes mais efetivo em detectar lesões cervicais destinadas a se tornarem invasivas, do que se realizado aos 20 anos de idade, e 10 vezes mais efetivo do que quando realizado aos 25 anos de idade. Além disso, deve-se considerar, apesar de não ser essa a justificativa presente nos estudos, o caráter iatrogênico que o teste de Papanicolaou pode assumir se for oferecido às mulheres jovens, sem risco epidemiológico, levando ao aumento no número de exames falso-positivos, que para serem desmentidos teriam necessidade da adoção de outros procedimentos mais invasivos.

Têm-se priorizado também a periodicidade trienal do exame colpocitológico, baseado em estudos que sugerem que não há diferenças significativas na redução da incidência do câncer cervical quando se realizam exames com intervalos anuais ou trienais. Por exemplo, estudos de coorte e de caso-controle realizados em diferentes países mostram que o rastreamento de $100 \%$ da população feminina de 35 a 64 anos, anualmente ou com intervalos trienais, apresenta resultados semelhantes quanto à redução na incidência do câncer cervical, 93,5\% e 91,4\%, respectivamente.7 No entanto, os custos para um programa de rastreamento anual seriam bem mais elevados devido ao maior número de coletas de material cérvico-vaginal exigidas, ferindo, portanto, a lógica custo-benefício que permeia as políticas de saúde, principalmente em países com escassez de recursos, como no caso brasileiro. Como mostrou Day, 7 o total de exames necessários para cobrir uma população, por exemplo, de 100.000 mulheres anualmente seria de três milhões, ao passo que para cobrir a mesma população, porém num intervalo trienal, o custo da ação seria reduzido para um terço deste total de exames.

Sob a lógica epidemiológica do risco e da relação custo-benefício, a World Health Organization (WHO) 12 propõe, para países com recursos financeiros limitados, o rastreamento de lesões cervicais pelo menos uma vez na vida em todas as mulheres com idade ao redor dos 40 anos e à medida que se disponha de mais recursos, propõe-se rastrear lesões cervicais a cada 10 ou cinco anos em mulheres entre 35 e 55 anos de idade. Quando não há limitação de recursos, a WHO recomenda que a prevenção ideal do câncer de colo do útero seja feita anualmente, por dois anos consecutivos, a partir dos 25 anos de idade e, caso esses exames sejam negativos, a cada três anos até os 60 anos de idade. 12

A Tabela 1 sintetiza a análise comparativa realizada por Linos e Riza13 entre diferentes programas nacionais de screening conduzidos em alguns países europeus, apresentando suas principais similitudes e diferenças nas estratégias implantadas de screening. A Finlândia, por exemplo, foi um dos países europeus pioneiros na implementação de um programa organizado de screening de âmbito nacional, iniciado no começo da década de 60 , alcançando uma cobertura relativamente alta entre a população feminina pertencente à faixa etária de 30 a 60 anos, tendo grande impacto na redução das taxas de incidência por câncer cervical, como apontado por Gustafsson et al. ${ }^{6}$. 
Comparações entre os diferentes programas nacionais de screening de câncer cervical conduzidos em sete países europeus, 1963-1996.

\begin{tabular}{|c|c|c|c|c|}
\hline País & Ano de início & $\begin{array}{c}\text { Faixa etária } \\
\text { "alvo" }\end{array}$ & $\begin{array}{c}\text { Intervalo } \\
\text { recomendado* }\end{array}$ & Cobertura (\%) \\
\hline \multirow[t]{2}{*}{ Alemanha } & 1971 (ex - Ocidental) & $\geq 20$ anos & 1 ano & $46,0-50,0$ \\
\hline & 1991 (ex - Oriental) & & & \\
\hline Finlândia & 1963 & $30-60$ anos & 5 anos & 89,5 \\
\hline \multirow[t]{2}{*}{ França } & 1990 (quatro programas em & $25-65$ anos & 3 anos & $22,0-69,0$ \\
\hline & diferentes áreas) & & & \\
\hline \multirow[t]{2}{*}{ Inglaterra } & 1988 & $20-64$ anos & $3-5$ anos & 84,0 \\
\hline & 1980 (Florença) & & & \\
\hline \multirow[t]{2}{*}{ Itália } & 1992 (Turim) & $25-64$ anos & 3 anos & 74,0 \\
\hline & 1995 (restante) & & & \\
\hline Países Baixos & 1996 & $30-60$ anos & 5 anos & 80,0 \\
\hline Suécia & Metade dos anos 60 & $20-59$ anos & 3 anos & $50,0-70,0$ \\
\hline
\end{tabular}

* Após um teste negativo; com exceção da França, cujo intervalo trienal é seguido após dois testes anuais negativos

Fonte: Linos, Riza. Eur J Cancer 2000; 36: 2260-5.13

Observa-se na Tabela 1 que estes países seguem diferentes estratégias de screening, quanto à idade de início e término da coleta do material cérvicouterino e de sua periodicidade, sendo que algumas estratégias assemelham-se entre si, como os programas conduzidos por países como a França, Itália e Inglaterra que priorizam a faixa etária dos 20 ao 65 anos e a periodicidade trienal na realização do exame; outros países como a Finlândia e Países Baixos priorizam faixas etárias mais restritas, entre 30 e 60 anos, e intervalos mais longos entre os exames; e há ainda países, como a Alemanha, que adotam a periodicidade anual e convidam todas as mulheres a partir dos 20 anos de idade a participarem dos programas de screening, não estabelecendo uma idade limite para seu término. 13

Já em países como os Estados Unidos e Canadá, por meio de organizações médico-preventivas, como a American Cancer Society (ACS), a US Preventive Services Task Force (USPSTF) e a Canadian Task Force on Preventive Health Care (CTFPHC), as recomendações são menos restritivas quanto ao início da coleta em programas de screening. A ACS e a USPSTF recomendam o início da coleta aos 18 anos ou logo após a iniciação sexual; a ACS recomenda a periodicidade anual e após dois ou três testes negativos (normais), tal frequêencia pode ser reduzida sob critério médico e a USPSTF recomenda a periodicidade trienal do exame, após dois ou três testes anuais negativos. ${ }^{14}$ Ambas organizações norte-americanas recomendam ainda a descontinuidade da participação nos programas de screening após os 65 anos de idade, caso os testes anteriores tenham sido consistentemente negativos. ${ }^{14}$ A CTFPHC e vários painéis canadenses de consenso nacional, por sua vez, recomendam que a coleta de material cérvicovaginal para o teste de Papanicolaou se inicie aos 18 anos ou logo após a iniciação sexual, e após dois testes anuais negativos (normais), recomenda-se a periodicidade trienal até os 69 anos de idade, quando a coleta pode ser interrompida, caso não haja nenhum teste anterior alterado. 14

No Brasil, particularmente no estado de São Paulo, a introdução do teste de Papanicolaou ocorreu em meados da década de 70 e se ampliou com o surgimento do PAISM (Programa de Assistência Integral à Mulher) em 1983, que tinha como objetivos programáticos implantar ou ampliar as atividades 
de diagnóstico precoce do câncer cervical, promover ações educativas na prevenção da doença, estendendo, assim, a assistência à saúde da mulher para além dos limites do ciclo gravídico-puerperal. ${ }^{15}$ Em 1988, o Ministério da Saúde passou a seguir a recomendação da Organização Mundial da Saúde para que o teste de Papanicolaou fosse realizado a cada três anos em mulheres entre 25 e 60 anos de idade, após dois exames anuais negativos. Mais recentemente, em 1996, o Ministério da Saúde, em parceria com o Instituto Nacional do Câncer (INCA), implantou o programa Viva Mulher, de âmbito nacional, envolvendo cinco capitais brasileiras, tendo como população-alvo as mulheres pertencentes à faixa etária mais restrita, entre 35 e 49 anos. 16 Em 1998, foi realizada a fase de intensificação da coleta durante a Campanha Nacional de Combate ao Câncer CérvicoUterino.

Como visto, não há uma homogeneidade de ações e recomendações a respeito da melhor estratégia a ser seguida pelos programas de screening, cabendo aos diferentes países e regiões adaptá-las segundo as necessidades epidemiológicas e sociais de cada região e da disponibilidade de recursos financeiros. Mas, sob tais recomendações estabeleci- das pelos órgãos competentes, o que podemos falar da cobertura do teste de Papanicolaou no Brasil e no mundo, considerando as peculiaridades de cada região sócio-demográfica?

\section{Cobertura do teste de Papanicolaou: quem não faz e por quê?}

Se em países desenvolvidos o teste de Papanicolaou adquiriu o status de uma técnica de rastreamento efetiva e eficiente em reduzir as taxas de morbi-mortalidade por câncer cervical, o mesmo não foi observado nos países em desenvolvimento, cuja cobertura do teste ainda não alcançou níveis suficientes e coerentes com as necessidades da população feminina sob maior risco.

A Tabela 2 apresenta a prevalência na realização do teste de Papanicolaou observada em diferentes países norte-americanos, europeus e latinos, incluindo o Brasil, a partir de estudos transversais de base populacional e institucional, localizados em revisão da literatura compreendendo o período de 1989 a 1999. 17

Tabela 2

Prevalência na realização do teste de Papanicolaou em alguns países da América do Norte, América Latina e Europa, 1989 -1999.

\begin{tabular}{|c|c|c|c|}
\hline País & Método e amostra & $\begin{array}{l}\text { Realização do testede } \\
\text { Papanicolaou }\end{array}$ & Prevalência (\%) \\
\hline $\begin{array}{l}\text { Estados Unidos } \\
\text { Harlan et al.,18 }\end{array}$ & $\begin{array}{l}\text { Estudo transversal de base populacional } \\
\text { nacional }-12.868 \text { mulheres } \geq 18 \text { anos }\end{array}$ & Últimos três anos & $\begin{array}{l}\text { 73,3 (brancas); } \\
\text { 78,9 (negras); } \\
65,0 \text { (hispânicas) }\end{array}$ \\
\hline Estados Unidos & Estudo transversal de base populacional & Alguma vez na vida & 91,0 \\
\hline Kirkman-Liff, Kronenfeld, 19 & 3.100 adultos americanos e latinos & Últimos dois anos & 68,0 \\
\hline Estados Unidos & Estudo transversal de base populacional & Alguma vez na vida & 96,8 \\
\hline Bostick et al.20 & 2.726 mulheres entre 25 - 74 anos & Últimos dois anos & 80,9 \\
\hline $\begin{array}{l}\text { Estados Unidos } \\
\text { Wilcox e Mosher, } 21\end{array}$ & $\begin{array}{l}\text { Estudo transversal de base populacional } \\
\text { nacional - } 8.450 \text { mulheres entre } 15-44 \\
\text { anos }\end{array}$ & Último ano & 67,0 \\
\hline $\begin{array}{l}\text { Estados Unidos } \\
\text { Pérez-Stable et al.,22 }\end{array}$ & $\begin{array}{l}\text { Estudo transversal com amostra } \\
\text { aleatória de usuárias de serviços - } 714 \\
\text { latinas e americanas entre } 35-74 \text { anos }\end{array}$ & Últimos três anos & $\begin{array}{c}86,7 \text { (latinas) e } \\
88,4 \text { (americanas) }\end{array}$ \\
\hline
\end{tabular}




\begin{tabular}{|c|c|c|c|}
\hline País & Método e amostra & $\begin{array}{l}\text { Realização do testede } \\
\text { Papanicolaou }\end{array}$ & Prevalência (\%) \\
\hline Estados Unidos & Estudo transversal de base populacional & Últimos três anos & 89,0 \\
\hline Kottke et al.,23 & por telefone -1.019 mulheres $\geq 18$ anos & Último ano & 60,0 \\
\hline $\begin{array}{l}\text { Estados Unidos } \\
\text { Chavez et al.,24 }\end{array}$ & $\begin{array}{l}\text { Estudo transversal de base populacional } \\
\text { por telefone - } 1.225 \text { mulheres latinas e } \\
\text { americanas acima de } 17 \text { anos }\end{array}$ & Nos últimos três anos & $\begin{array}{c}\text { 89,6 (latinas); } \\
64,8 \text { (imigrantes); } \\
92,7 \text { (americanas) }\end{array}$ \\
\hline $\begin{array}{l}\text { Estados Unidos } \\
\text { McPhee et al., } 25\end{array}$ & $\begin{array}{l}\text { Estudo transversal em duas } \\
\text { comunidades com } 645 \text { mulheres } \\
\text { vietnamitas } \geq 18 \text { anos }\end{array}$ & Alguma vez na vida & 42,8 \\
\hline $\begin{array}{l}\text { Estados Unidos } \\
\text { O'Malley et al.,26 }\end{array}$ & $\begin{array}{l}\text { Estudo transversal de base populacional } \\
\text { por telefone - } 1.420 \text { mulheres entre } 18 \text { - } \\
74 \text { anos (sete grupos étnicos) }\end{array}$ & Alguma vez na vida & 74,0 a 94,7 \\
\hline $\begin{array}{l}\text { Estados Unidos } \\
\text { Wismer et al.,27 }\end{array}$ & $\begin{array}{l}\text { Estudo transversal de base populacional } \\
\text { por telefone }-818 \text { mulheres coreanas } \\
>17 \text { anos }\end{array}$ & $\begin{array}{l}\text { Alguma vez na vida } \\
\text { Últimos três anos } \\
\text { Último ano }\end{array}$ & $\begin{array}{l}63,0 \\
55,0 \\
34,7\end{array}$ \\
\hline Estados Unidos & Estudo transversal de base populacional- & Alguma vez na vida & 95,0 \\
\hline Risendal et al.,28 & $\begin{array}{l}519 \text { mulheres indio-americanas }>18 \\
\text { anos }\end{array}$ & Últimos três anos & 76,1 \\
\hline Estados Unidos & Estudo transversal de base populacional & Últimos três anos & 80,7 \\
\hline Simões et al.,29 & por telefone -1.609 mulheres $\geq 18$ anos & Último ano & 63,2 \\
\hline Estados Unidos & Estudo transversal de base populacional & Alguma vez na vida & 87,3 \\
\hline Mandelblatt et al.,30 & $\begin{array}{l}\text { por telefone - } 1.420 \text { mulheres negras e } \\
\text { hispânicas entre } 18-74 \text { anos }\end{array}$ & Últimos três anos & 62,0 \\
\hline $\begin{array}{l}\text { Estados Unidos } \\
\text { Zambrana et al., } 31\end{array}$ & $\begin{array}{l}\text { Estudo transversal de base populacional } \\
\text { nacional - } 2.391 \text { mulheres hispânicas } \\
\text { acima de } 17 \text { anos }\end{array}$ & Últimos três anos & 77,0 \\
\hline $\begin{array}{l}\text { Estados Unidos, Canadá } \\
\text { Katz e Hofer, } 32\end{array}$ & $\begin{array}{l}\text { Estudo transversal de base populacional } \\
\text { nacional - } 47.453 \text { mulheres entre } 18-74 \\
\text { anos }\end{array}$ & Últimos dois anos & $\begin{array}{l}\text { 74,0 (Canadá); } \\
\text { 76,4 (Estados } \\
\text { Unidos) }\end{array}$ \\
\hline $\begin{array}{l}\text { Canadá } \\
\text { Camirand et al., } 33\end{array}$ & $\begin{array}{l}\text { Estudo transversal de base populacional } \\
\text { com } 1.799 \text { mulheres entre } 18-35 \text { anos }\end{array}$ & $\begin{array}{l}\text { Alguma vez na vida } \\
\text { Último ano }\end{array}$ & $\begin{array}{l}88,1 \\
60,4\end{array}$ \\
\hline $\begin{array}{l}\text { Itália } \\
\text { Ronco et al.,34 }\end{array}$ & $\begin{array}{l}\text { Estudo transversal com amostra } \\
\text { aleatória de } 581 \text { usuárias de serviços } \\
\text { entre } 18-69 \text { anos }\end{array}$ & $\begin{array}{l}\text { Alguma vez na vida } \\
\text { Últimos três anos }\end{array}$ & $\begin{array}{l}52,8 \\
40,9\end{array}$ \\
\hline $\begin{array}{l}\text { Inglaterra } \\
\text { Schwartz et al., } 35\end{array}$ & $\begin{array}{l}\text { Estudo transversal com amostra } \\
\text { aleatória de } 600 \text { usuárias de serviços } \\
<65 \text { anos }\end{array}$ & Alguma vez na vida & 77,0 \\
\hline
\end{tabular}




\begin{tabular}{|c|c|c|c|}
\hline País & Método e amostra & $\begin{array}{l}\text { Realização do testede } \\
\text { Papanicolaou }\end{array}$ & Prevalência (\%) \\
\hline \multirow{3}{*}{$\begin{array}{l}\text { México } \\
\text { Lazcano-Ponce et al., } 36\end{array}$} & Estudo transversal de base populacional- & Alguma vez na vida & 64,2 (urbana) \\
\hline & 4.208 mulheres entre $15-49$ anos & & 30,0 (rural) \\
\hline & & Último ano & 75,0 (urbano-rural) \\
\hline México & Estudo transversal com amostra & Últimos três anos & 68,6 \\
\hline Cancio et al., 37 & $\begin{array}{l}\text { aleatória de } 746 \text { usuárias de serviços } \\
\text { entre } 28 \text { - } 65 \text { anos }\end{array}$ & Último ano & 41,4 \\
\hline México & Estudo transversal de base populacional- & Alguma vez na vida & 77,5 \\
\hline Hernandez-Hernandez et al., 38 & 1.215 mulheres entre $18-74$ anos & Últimos três anos & 35,5 \\
\hline Brasil (São Paulo) & Estudo transversal com amostra & Alguma vez na vida & 54,6 - 60,9 (último \\
\hline \multirow[t]{2}{*}{ Pinotti et al.,39 } & aleatória de 3.703 usuárias de serviços & & ano) \\
\hline & públicos de saúde entre 15 a 49 anos & & \\
\hline Brasil & Estudo transversal de base populacional- & Alguma vez na vida & 64,0 \\
\hline Lopes et al., 40 & 1478 mulheres acima de 16 anos & & \\
\hline Brasil (São Paulo) & Estudo transversal de base populacional- & Alguma vez na vida & 68,9 \\
\hline \multirow[t]{2}{*}{ Nascimento et al., 41} & 967 mulheres entre $15-59$ anos & Últimos três anos & 60,8 \\
\hline & & Último ano & 41,3 \\
\hline Brasil (Rio Grande do Sul) & Estudo transversal com amostra de 224 & Alguma vez na vida & $83,4 \%(72,2$ \\
\hline Karam et al., 42 & usuárias de serviços entre 15 - 55 anos & & último ano) \\
\hline Brasil (São Paulo) & Estudo transversal com amostra de 135 & Alguma vez na vida & 68,9 \\
\hline Merigui et al.,43 & usuárias de serviços > 15 anos & & \\
\hline Brasil (Rio Grande do Sul) & Estudo transversal de base populacional & Últimos três anos & 65,0 \\
\hline Dias da Costa et al., 44 & 934 mulheres entre $20-69$ anos & & \\
\hline Brasil (São Paulo) & Estudo transversal de base populacional & Alguma vez na vida & 86,0 \\
\hline Pinho45* & 1.046 mulheres de 15 - 49 anos & Nos últimos três anos & 77,0 \\
\hline
\end{tabular}

* Estudo referente à dissertação de mestrado concluída em 2002 e não localizada na revisão bibliográfica de artigos publicados no período de 1989-1999.

Embora haja diferenças metodológicas entre os estudos, quanto à representatividade da amostra, faixa etária das mulheres entrevistadas e do período investigado na realização do teste de Papanicolaou, se pelo menos uma vez na vida, nos últimos três anos ou no último ano, anteriores à entrevista, pode-se abstrair, resumidamente, desta tabela algumas observações gerais a respeito da cobertura do teste de Papanicolaou entre as diferentes populações investigadas.

Observa-se que há uma predominância de estudos realizados em países desenvolvidos e poucos es- tudos realizados em países em desenvolvimento, justamente a região onde se concentram os casos de câncer de colo do útero. Em países como os Estados Unidos a prevalência na realização do teste de $\mathrm{Pa}$ panicolaou alguma vez na vida alcançou de $43 \%$ a 97\%, sendo consideravelmente menor entre as minorias étnicas residentes nos EUA, como as mulheres vietnamitas, coreanas e indo-americanas e aquelas de origem latina ou hispânica, cuja cobertura do teste tem se mostrado inferior à cobertura entre as mulheres de origem anglo-saxônica. ${ }^{18-31}$ No Canadá, a cober- 
tura do teste de Papanicolaou alguma vez na vida e nos últimos dois anos anteriores à entrevista, observada em estudos de base populacional, foi muito próxima àquela observada entre a população feminina americana. 32,33 Em estudos realizados em países europeus, como a Itália e Inglaterra, com a população usuária de serviços de saúde, a prevalência observada na realização do teste alguma vez na vida foi de $53 \%$ entre usuárias italianas, 34 e de $77 \%$ entre usuárias inglesas. 35

Nos países latinos, foram relatadas prevalências mais baixas na realização do teste de Papanicolaou pelo menos uma vez na vida, como na zona rural de Oaxaca, no México (30\%).36 Em outras localidades de características urbano-rural a prevalência na realização do teste esteve ao redor de $70 \%$ da população entrevistada, ${ }^{36-38}$ aproximando-se da cobertura observada entre mulheres de origem latina ou hispânica e pertencentes às minorias étnicas residentes nos EUA.

No Brasil, seis estudos foram identificados no período de 1989 a $1999,39-44$ sendo que apenas três foram realizados com uma amostra aleatória de base populacional (Tabela 2). Particularmente no município de São Paulo, em 1987, foi realizado um estudo transversal de base populacional sobre a cobertura do teste de Papanicolaou em que os autores estimaram uma prevalência na realização do teste alguma vez na vida de $68,9 \%$ entre a população feminina de 15 a 49 anos, ${ }^{41}$ muito acima daquela estimada para o estado de São Paulo pelos órgãos oficiais, em que menos de $20 \%$ da população feminina de 25 a 59 anos estaria coberta pelo teste. 5 Mas, vale destacar, que fora do período investigado, o último inquérito epidemiológico realizado no município de São Paulo data de 2002, com uma amostra aleatória e representativa da população feminina de 15 a 49 anos, em que se observou uma prevalência na realização do teste de Papanicolaou de $86 \%$ alguma vez na vida e $77 \%$ nos últimos três anos, excluídas as mulheres que nunca tiveram atividade sexual. 45

A discrepância de tais resultados com a cobertura estimada pelos órgãos oficiais pode ser atribuída ao fato destas estimativas serem baseadas no número total de exames realizados anualmente pelo Sistema Único de Saúde (SUS), não considerando, portanto, o intervalo trienal entre os exames; não permitirem distinguir as mulheres que fizeram vários exames daquelas que fizeram apenas um teste; a inclusão de mulheres acima de 15 anos, sem dados sobre início da atividade sexual e, principalmente, por não considerarem os exames colpocitológicos realizados em serviços privados ou conveniados fora do Sistema Único de Saúde.
Os poucos dados provenientes de estudos epidemiológicos realizados com uma amostra representativa da população feminina brasileira sugerem que além da cobertura ser relativamente deficiente, ela está distribuída desigualmente entre a população usuária, com a realização do teste concentrando-se, geralmente, entre os grupos de menor risco para o câncer cervical.40,41,44,45

Os dados brasileiros sobre a cobertura do teste de Papanicolaou, em conjunto àqueles provenientes de outros países da América Latina e de países desenvolvidos, como EUA e Canadá, apresentados anteriormente, sugerem que há diferenças no acesso e utilização do teste de Papanicolaou entre a população feminina, e que estas são mais pronunciadas para determinados subgrupos populacionais e, geralmente, descompassadas com as necessidades epidemiológicas da população usuária. Além disso, tais diferenças persistem, inclusive, em países desenvolvidos, cuja cobertura global do teste de Papanicolaou é relativamente alta, aproximando-se daquela preconizada pela Organização Mundial de Saúde, segundo a qual uma cobertura de $85 \%$ já produziria algum impacto no quadro epidemiológico do câncer cérvico-uterino. 12

$\mathrm{Na}$ tentativa de identificar as origens dessas diferenças no acesso e utilização do teste, vários estudos epidemiológicos, majoritariamente norteamericanos, têm caracterizado o perfil das usuárias do teste de Papanicolaou, sendo proposto um modelo explicativo de cunho muito mais individual, atribuindo aos indivíduos, neste caso as mulheres, a responsabilidade pela insuficiente ou inadequada utilização de tais serviços preventivos. Assim, a realização do teste de Papanicolaou passaria a ser dependente de um conjunto de características individuais das usuárias, concentrando-se em aspectos sócio-demográficos, entre eles, a idade, o estado civil e a escolaridade e renda como preditores do acesso diferenciado que mulheres de maior nível socioeconômico teriam à assistência médico-preventiva. ${ }^{41,46-48}$

Ainda sob este modelo destacam-se, nesses estudos, os aspectos sexuais e reprodutivos, como início da vida sexual, número de gestações na vida e uso de métodos contraceptivos, em que a realização do teste de Papanicolaou aconteceria em conjunção a atividades de assistência ginecológica, obstétrica ou de planejamento familiar. 21,38,49

Outros fatores como as crenças e atitudes em relação ao câncer cérvico-uterino e ao exame colpocitológico,28,50,51 e a auto-percepção da severidade e suscetibilidade à doença também têm sido descritos como elementos explicativos de base individual. $52-54$ Sob tais aspectos, alguns estudos se valem do "mode- 
lo de crenças em saúde", formulado por Becker e Rosenstock 55 , que enuncia que o comportamento preventivo em saúde é decorrente da presença de quatro tipos diferentes de crenças dos indivíduos: percepção da severidade da doença, de sua susceptibilidade, dos benefícios/eficácia da ação preventiva e das barreiras a essa ação. No caso específico do câncer cérvico-uterino e da realização do teste de Papanicolaou, esses quatro tipo de crenças têm sido investigadas a partir de uma escala de concordâncias a afirmativas como: o câncer é uma doença séria que pode afetar qualquer pessoa, a concepção do câncer como uma doença fatal e incurável (severidade da doença); a preocupação em adquirir a doença, a percepção de estar sob risco ou de que o câncer atinge somente um grupo específico de mulheres (susceptibilidade à doença); a crença de que o câncer não pode ser curado mesmo sendo detectado precocemente, que não há muito o que fazer para preveni-lo e que o teste de Papanicolaou não é efetivo em detectá-lo (benefícios percebidos da ação); a preferência por não saber que tem a doença, o custo do teste, o desconforto e a vergonha associados ao procedimento (barreiras percebidas à ação). 51,52

É necessário fazer, contudo, algumas ressalvas quanto ao uso do "modelo de crenças em saúde" em estudos epidemiológicos para explicar o que é chamado de comportamento preventivo em saúde. Neste modelo, tem-se como pressuposto que tal comportamento é dependente de um conjunto de crenças dos indivíduos, limitado à esfera psicossocial e, portanto, negligenciam-se outros fatores responsáveis pelo comportamento em saúde, como a própria posição sociocultural, política e econômica das mulheres e a disponibilidade e acesso aos serviços de saúde que oferecem o serviço preventivo. A ausência de tais considerações no "modelo de crenças em saúde" pode explicar, em parte, a variabilidade de resultados encontrados nos estudos epidemiológicos que utilizaram tal modelo, não sendo unânime a presença de associações entre as variáveis desse modelo e a realização do teste de Papanicolaou. O mesmo pode se falar sobre os estudos que, pautados em discursos comportamentalistas sobre estilos de vida, destacam outras ações de caráter preventivo como preditoras na realização do teste de Papanicolaou, entre elas, o auto-exame de mamas, a realização de mamografia, a prática de exercício físico e o hábito de não fumar.29,31,34 Segundo os autores destes estudos, as mulheres adeptas a tais atitudes e comportamentos preventivos à saúde se engajariam com maior facilidade em outras ações preventivas como a realização periódica do teste de Papanicolaou. No entanto, a utilização de uma perspectiva de estilos de vida pode ser problemática do mesmo modo que a utilização do modelo de crenças em saúde, pois têmse como pressuposto que o indivíduo possui total responsabilidade para com sua saúde, suas "escolhas" comportamentais e gerenciamento e modificação de suas condutas individuais de risco. Portanto, qualquer falha em sua saúde é devido à "negligência" ou ao comportamento inadequado, não saudável, ou de risco dos indivíduos. Uma situação um tanto paradoxal, como coloca Castiel pois, segundo ele, "... se são atribuidas às pessoas suas escolhas de estilos de vida (dentro, é claro, de suas margens de aquisição/acesso), incluidos no "pacote" fatores considerados de risco, então, supõe-se este subconjunto como o estilo de risco, como se as pessoas "escolhessem" exposições a riscos como forma de levar suas vidas" (1999: 60).56 Logo, segundo o autor, não teria sentido a aplicação desse conceito em condições socioeconômicas desfavoráveis, em que as pessoas não elegem estilos de vida, pois na falta de opções, o que lhes sobram são "estratégias de sobrevivência". 56

Enfim, essas características e comportamentos individuais agiriam, segundo os modelos explicativos presentes, como facilitadores ou como barreiras de acesso aos serviços de saúde. Porém, o conceito de acesso na maioria desses estudos restringese ao plano individual de análise, ou seja, o acesso ao serviço de saúde é dependente de características e comportamentos dos indivíduos que demandam por serviços de cuidado à saúde e não consideram outros planos de análise de acesso, como aqueles definidos por Puentes-Markides: 57 O plano institucional ou organizacional, que investigue a presença de fatores relativos à organização da assistência à saúde da mulher, quanto aos programas de prevenção e controle do câncer cérvico-uterino, à qualidade $\mathrm{e}$ cobertura do teste de Papanicolaou e às relações estabelecidas entre instituição/médico-paciente, e o plano social, ou seja, o estilo de desenvolvimento do país, as políticas econômicas e de saúde pública, o status da mulher na sociedade e as relações de desigualdades sociais no acesso e utilização dos serviços de saúde e que estão organizadas ao longo de no mínimo três eixos: classe, raça/etnia e gênero.

Nos poucos estudos que abordam as dificuldades de acesso e realização do teste de Papanicolaou sob um aspecto organizacional/programático, essa análise limita-se ao estudo das dificuldades financeiras relacionadas ao custo da assistência ou as dificuldades de acessibilidade geográfica ao serviço de saúde, traduzidas em problemas e custos de transporte ou na localização dos serviços.29,30,35,38 Porém, tais dificuldades parecem ser de menor im- 
portância em relação a outras barreiras de caráter organizacional ou logístico, como longo período de espera para ser atendida ou marcar uma consulta, disponibilidade de recursos materiais e humanos, expressos na ausência de instrumentos, absenteísmo médico, falta de vagas em consultas, falta de tempo devido à carga horária de trabalho da usuária ou não ter com quem deixar os filhos, ou os problemas na relação médico/instituição-paciente. 48

Quanto à utilização de uma dimensão de cunho social para explicar o acesso e a utilização do teste de Papanicolaou, quando presente, remete às questões de desigualdades sociais entre classe social e raça/etnia, mas omite a existência de desigualdades de gênero verificadas na utilização dos serviços de saúde, como também, na reprodução dessas desigualdades no atendimento oferecido pelos serviços de saúde às necessidades das mulheres.

As desigualdades de raça/etnia na realização do teste de Papanicolaou presentes num modelo explicativo remetem à questão do acesso (no plano individual) restrito que as mulheres de determinada raça ou etnia têm no sistema de saúde, principalmente as mulheres latinas e asiáticas imigrantes, visto que a maior parte dos estudos é realizada nos Estados Unidos. A baixa cobertura entre esses grupos populacionais têm sido explicada pelo acesso diferenciado que eles têm aos serviços de saúde em geral e, em particular, aos serviços preventivos, dependente de características sócio-demográficas e culturais, como o tempo de residência nos Estados Unidos, o nível socioeconômico, geralmente muito baixo entre esses grupos, as barreiras de língua, a falta de seguro-saúde, entre outras.31,50,58,59 Enfatizam ainda a relação da etnia com crenças e atitudes negativas em relação ao câncer e ao teste de Papanicolaou, sendo que para alguns autores as crenças fatalísticas perante a doença ou ao teste seriam próprias das mulheres de origem latina ou indo-asiática, 24,59 e não produzidas e reforçadas dentro de um contexto social, políticoeconômico restritivo e reprodutor de desigualdades no acesso e utilização de serviços preventivos entre as populações imigrantes.

É escassa, portanto, qualquer avaliação mais consistente, sobretudo nacional, que incorpore ou, ao menos, considere outros elementos que potencialmente possam explicar o acesso aos serviços preventivos, principalmente aqueles elementos relacionados ao contexto institucional e social do qual a usuária faz parte. Pensando nisso, propõe-se analisar o acesso e a utilização de serviços preventivos, como o teste de Papanicolaou, sob a égide de um modelo "contextual", composto por três planos: individual, organizacional/programático e social, dispostos numa estrutura dinâmica e relacional, sendo proposto que a realização do teste de Papanicolaou é produto da interação desses três planos e que neles estariam presentes os elementos necessários para definir uma situação de vulnerabilidade individual, programática/organizacional e social - conceito originalmente utilizado no campo das investigações sobre a infecção ao HIV/AIDS, particularmente por Mann et al. $60 \mathrm{e}$ Ayres et al.61, sendo, aqui, emprestado e adaptado devido a sua adequação à abordagem do fenômeno em questão - ao câncer de colo do útero e ao acesso e utilização restrito às práticas preventivas a essa doença.

\section{Modelo "contextual" para analisar o acesso e utilização do teste de Papanicolaou}

Neste modelo, representado pela Figura 1, ter-se-ia um plano individual constituído por um conjunto de características das usuárias relacionadas à realização do teste de Papanicolaou, já descritos anteriormente por outros autores, porém reorganizados em torno do conceito de vulnerabilidade individual.60,61 O modelo de crenças em saúde, por exemplo, poderia ser reformulado sob este conceito de vulnerabilidade imposta aos indivíduos ou criada e percebida por eles a partir de um conjunto de fatores cognitivos relacionados à real falta de conhecimento científico sobre a doença e o teste de Papanicolaou. De fato o acesso restrito à informação e à sua correta assimilação, ou o não reconhecimento da susceptibilidade à infecção pelo vírus Papiloma Humano (HPV) em relações sexuais desprotegidas, diminuem a probabilidade de busca a práticas de prevenção secundária ao câncer de colo uterino. Esta vulnerabilidade individual também é (re)criada e percebida nas relações interpessoais e coletivas, no compartilhamento de experiências de vida pessoais ou de outras pessoas conhecidas, ou em resposta ao que alguns autores chamam de "crenças normativas", 62 ou seja, as percepções criadas pelos indivíduos sobre comportamentos que deveriam ser seguidos segundo as normas preditas pela sociedade. 
Modelo teórico dos fatores associados ao acesso e utilização do teste de Papanicolaou.

Acessibilidade/ Disponibilidade Planejamento e organização de ações preventivas e promotoras em saúde Continuidade do cuidado

Sistema de informação eficiente para acompanhamento das ações

Recursos humanos e materiais

Programas de prevenção e controle efetivos,

não interferência na autonomia individual e

abordagem integral do sujeito-mulher
Plano Social

Nível de desenvolvimento do país

Políticas públicas na área de saúde da mulher, recursos financeiros; Condição e posição socioeconômica da mulher na sociedade, desigualdades nas relações de gênero, raça, classe social, geração, etc. Violência social/estrutural; Valores culturais e Normas sociais

Plano institucional Plano de Plano programático

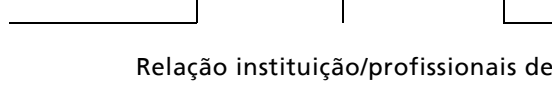
saúde - usuários

Acolhimento/Qualidade da atenção Aceitação/grau de satisfação/Resolutividade Violência Institucional (locus de maus-tratos e humilhação)

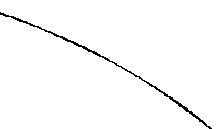


vários estudos têm mostrado que sentimentos de medo em relação ao teste, à doença, à dor do exame ginecológico e ao recebimento de um resultado positivo são motivos comuns para a não realização do teste de Papanicolaou. 22,40,43,46,49,52

Tendo em vista o exposto, qualquer tentativa que vise a uma mudança de comportamento em direção a práticas preventivas tomada somente no plano individual é falha, pois tal mudança não depende, exclusivamente, de um sistema de crenças individuais/privadas, mas de toda uma complexa rede de relações interpessoais, organizacionais ou programáticas que tem ligação com as estratégias discursivas e com as técnicas utilizadas pelos programas em prevenção ao câncer e aos aspectos estruturais relacionados aos facilitadores e as barreiras sociais, culturais, econômicas e políticas relacionadas ao comportamento preventivo. No caso específico do câncer de colo do útero, a ênfase, por exemplo, de estratégias de prevenção primária à doença como o uso de preservativos femininos e masculinos na prevenção à infecção por HPV, embora seja amplamente desejável e diminua potencialmente o risco de infecção desse tipo de infecção65 - ainda que alguns estudos sugiram que o uso freqüente de condom não apresente um efeito cem por cento protetor, devido aos casos de infecção por HPV em áreas genitais não protegidas pelo condom66 - seu uso está sujeito as mesmas condições acima estabelecidas para a realização do teste de Papanicolaou que definem uma situação de vulnerabilidade individual, ou seja, o grau de conhecimento a respeito da doença e formas de prevenção, a percepção de risco à infecção, as crenças normativas, as barreiras culturais, as iniquidade de gênero e como estas se revelam no grau de autonomia e poder de negociação sexual nas relações afetivas-sexuais, o acesso aos serviços de saúde e a oferta de preservativos. 67

Ainda no plano individual, sob este novo modelo contextual, ter-se-ia também a incorporação de outros elementos antes não pensados e contribuintes para uma situação de vulnerabilidade individual, como o papel da violência contra a mulher, especialmente aquela perpetrada no âmbito doméstico, por familiares ou parceiros, cujas repercussões para a saúde das mulheres e para o uso que fazem dos serviços vêm sendo recentemente estudadas. A violência física e sexual parece aumentar o risco das mulheres para problemas ginecológicos, além de influenciar o uso de serviços de saúde.68,69 A dinâmica da interação entre mulheres em situação de violência e a utilização de serviços de saúde em geral e, em particular, de serviços preventivos é complexa e ainda pouco estudada, e talvez possa depender do tipo de violência sofrida, de sua severidade e do perpetrador do ato violento, ressaltando que todas as formas de violência têm conseqüências que ultrapassam a esfera da saúde física, tendo importantes repercussões para a saúde mental das mulheres e são formas, potencialmente, incapacitantes do cuidado de si e de outros, que poderia ser expressa em uma busca diminuída por cuidados preventivos em saúde. No caso específico da situação de violência sexual, essa pode acabar restringindo a busca por cuidados médicos em geral e específicos, como uma consulta ginecológica, por medo, vergonha ou medo de retaliação por parte do parceiro por constituir-se numa ameaça ao desvelamento da violência sofrida. $\mathrm{Ou}$ ainda, a busca por cuidados ginecológicos entre mulheres que viveram situações de violência sexual pode estar dificultada por constituir-se num espaço de revivescência da experiência dolorosa do episódio de violência, principalmente se o exame ginecológico for realizado sem explicação do procedimento e do seu sentido, de forma fria e descuidada. Dessa forma, ao invés de constituir-se num espaço de acolhimento à mulher, a consulta ginecológica pode potencializar o sentimento de medo e rejeição ao exame. Esta relação entre a experiência de ter passado por uma situação de violência sexual e a realização do teste de Papanicolaou pôde ser vista no estudo de Pinho, ${ }^{45}$ em que a autora observou que as mulheres que haviam sofrido violência sexual por algum parceiro íntimo atual ou anterior tiveram uma probabilidade menor de terem realizado o teste de Papanicolaou.

Quanto ao plano organizacional ou programático (Figura 1), nesse encontrar-se-iam as características relacionadas à disponibilidade de serviços de saúde em relação à demanda, sua acessibilidade geográfica; à organização e às necessidades da população usuária, e ao planejamento de ações efetivas na prevenção e promoção à saúde, sob a forma de programas organizados de rastreamento do câncer cervical que primem pela não interferência na autonomia individual e pela abordagem integral do sujeito mulher. Todos esses elementos reorganizados em torno do conceito de vulnerabilidade organizacional ou programática, 60,61 os quais estariam expostas as mulheres que não dispusessem de adequado acesso e utilização do teste de Papanicolaou segundo as características presentes no plano organizacional ou programático.

Nesse plano, seria necessário também agregar outras dimensões de acesso pouco investigadas nos estudos, como por exemplo, a continuidade do cuidado, que tem sido sugerida por alguns autores como um importante preditor para a realização do teste de Papanicolaou, sendo caracterizada pela 
disponibilidade e utilização de um local usual de assistência e/ou a presença de um médico regular. 26 Ademais, a falta de continuidade do cuidado, dificultando a oferta de uma assistência contínua, prejudicaria a relação médico-paciente, o seu fortalecimento, o estabelecimento de confiança e a adesão das pacientes às práticas curativas e preventivas.

No caso específico de lesões cervicais, a falta de continuidade do cuidado e resolutividade do problema demandado são observadas, por exemplo, na falha no seguimento regular das usuárias, com ou sem resultados positivos de alterações cervicais, e no acesso e provisão de ações terapêuticas eficazes para as lesões detectadas. Sob este aspecto, tem-se como exemplo o não recebimento do resultado do teste realizado e o que é pior, sua distribuição desigual entre a população usuária, visto que o não recebimento parece ser mais comum entre as mulheres com menor nível socioeconômico, 40 o que pode refletir também a falta de acessibilidade ao serviço de saúde, particularmente em serviços públicos de saúde, cuja clientela majoritária pertence às classes socioeconômicas menos favorecidas. 36 Ademais, o não recebimento ou atraso do resultado do teste de Papanicolaou pode contribuir para a geração de crenças relacionadas ao mesmo e interferir na credibilidade e no grau de adesão das mulheres aos serviços e programas de prevenção em saúde. $70 \mathrm{O}$ não recebimento do resultado do teste ou o modo como ele é comunicado, se pessoalmente ou não, pode representar uma oportunidade perdida de desconstruir crenças e atitudes negativas em relação ao teste, sua finalidade, o significado de seus resultados, bem como em relação ao câncer cervical. Em direção à diminuição desta vulnerabilidade programática/organizacional, a qual estariam expostas as mulheres, uma proposta seria o planejamento de atividades assistenciais voltadas para "desmonopolização" do conhecimento restrito à esfera médica e a participação mais ativa das mulheres em relação a sua saúde, a partir, por exemplo, de atividades cada vez mais comuns em serviços de atenção primária, como os "grupos de Papa", nos quais são discutidos com as usuárias os resultados do teste de Papanicolaou realizado, o significado de um teste positivo ou negativo e dos achados bacteriológicos e sua relevância, a importância da periodicidade do teste, questões de higiene íntima e formas de prevenção às doenças sexualmente transmissíveis e ao HIV/AIDS, respeitando, é claro, a privacidade e o sigilo do resultado do teste caso, assim, seja a vontade das mulheres.

Ainda no plano programático/organizacional, no acesso e utilização do teste de Papanicolaou deve ser resguardada também a sua efetividade quanto à acuidade diagnóstica. Esse Teste é considerado um método altamente sensível, ou seja, sua precisão em diagnosticar corretamente os casos verdadeiros de lesões cervicais pré-neoplásicas e neoplásicas tem sido descrita entre $75 \%$ a mais de $90 \%$ dos casos. 71,72 Isto quer dizer que o teste será raramente negativo na presença da doença, resultando numa baixa taxa de testes falso-negativos. No entanto, segundo alguns autores, os resultados falso-negativos são responsáveis por cerca de $10 \%$ dos casos de câncer invasivo que progrediram a partir de lesões pré-neoplásicas não detectadas em testes anteriores, ${ }^{73}$ cuja confirmação diagnóstica não foi realizada a partir de uma nova coleta de material cérvico-vaginal num intervalo adequado. Tais resultados falso-negativos são, atualmente, uma das causas mais comuns de litígio médico envolvendo os screening de câncer de mama e de colo uterino, especialmente entre a população feminina americana e inglesa. ${ }^{74}$

Apesar do sistema de screening configurar-se, à primeira vista, como um processo simples, ele envolve um imbricado sistema de procedimentos clínicos e laboratoriais, sob responsabilidade de vários indivíduos, que vai desde a obtenção adequada e representativa do esfregaço cérvico-vaginal, passando pela preparação da lâmina, coloração e identificação, até a leitura e interpretação acurada do esfregaço. Logo, esse sistema, ao contrário do que se imagina, é um processo altamente especializado, dependente da competência laboratorial e maestria profissional. Alguns autores defendem a opinião de que a maioria das falhas no sistema de screening é decorrente dos esforços não realistas para fornecer um procedimento altamente especializado, mas com baixo custo, devido ao objetivo de torná-lo acessível à população feminina, o que se tornaria, financeira $\mathrm{e}$ tecnicamente, inviável para muitos laboratórios, além de ameaçar a acuidade diagnóstica do teste de Papanicolaou. 75

A dimensão organizacional/programática de acesso ao cuidado e ao serviço de saúde em interface com as características de acesso do ponto de vista individual constitui o que chamamos, aqui, de subplano de interação (contato), como podemos observar na Figura 1. É nesse plano, representado pela relação que se estabelece entre instituição/profissionais de saúde e usuárias, resultado da interação entre ca-racterísticas individuais e aquelas relacionadas aos serviços e programas em atenção à saúde, que se caracteriza a qualidade técnica e humana do cuidado, a arte do cuidado - o acolhimento - o grau de aceitação/satisfação do usuário ao serviço prestado e o grau de resolutividade ao problema demandado. 57,76 
No caso específico do exame ginecológico, com ou sem a coleta de material cérvico-vaginal, sentimentos de desconforto físico e psicológico são comuns entre relatos de mulheres que o realizam, 35,48,52,54 e grande parte destes sentimentos são originários de experiências prévias negativas de maus-tratos ou humilhação sofridas pelas mulheres durante o procedimento, realizado sem explicação do seu significado, de forma fria e descuidada, minando qualquer possibilidade de criação de um espaço de auto-conhecimento do corpo e da sexualidade da mulher. ${ }^{77}$ Há que se considerar a importância que se reveste a presença de maus-tratos ou humilhação em serviços de saúde em geral e, em particular, no atendimento ginecológico, na geração de um sentimento de insatisfação, de perda de confiança, de desrespeito e de subjugação de crenças e valores, levando, muitas vezes, à rejeição de certas práticas e técnicas assistenciais, como o exame pélvico e a realização do teste de Papanicolaou.

Este desconforto físico e psicológico reflete as relações historicamente estabelecidas entre as usuárias e os profissionais de saúde durante as ações de intervenção técnica, baseadas na visão tradicional de assistência à saúde das mulheres, traduzida em ações medicalizadas e apartadas de uma visão integral da mulher, cujas necessidades vão além dos aspectos físicos do corpo feminino, fazendo-se presentes no corpo social dessas mulheres. Portanto, são bem-vindas as estratégias de modificação de conduta/postura profissional frente à prática clínicoginecológica, que busquem traduzir o conceito de integralidade presente, originalmente, nas diretrizes do PAISM, enfatizando, segundo Osis "... não só a integração do colo, do útero e das mamas, mas também de outros aspectos não físicos da vida das mulheres. O que as mulheres defendem é que o integral se refira também ao contexto social, psicológico e emocional das mulheres a serem atendidas" (1998: 29). 78

Isoladamente ou em interação, os planos individual e organizacional/programático são consistentemente influenciados por outros elementos permissivos ou restritivos do plano social (Figura 1) relacionados ao nível de desenvolvimento socioeconômico do país; à existência de políticas públicas efetivas em saúde e à distribuição de recursos financeiros destinados a essa área; à condição e posição social das mulheres; às desigualdades nas relações sociais de gênero, raça, classe social, geração, etc., e seus impactos na saúde e no cuidado à saúde; aos valores culturais e normas sociais sobre o corpo, à sexualidade e à reprodução. Em suma, todos esses elementos potencialmente constituintes do que seria caracterizado pela vulnerabilidade social, 60,61 aos quais estariam expostas as mulheres.

Em direção à diminuição desta vulnerabilidade social, particularmente àquela relacionada às desigualdades nas relações sociais de gênero, torna-se essencial a sensibilização dos serviços de saúde com a questão de gênero, já que as iniqüidades presentes no acesso e utilização dos serviços de saúde e na qualidade da assistência prestada são reflexos também das desigualdades de poder nas relações de gênero dentro e fora da instituição médica, particularmente na instituição familiar. Tais desigualdades constituem-se um elemento restritivo ao exercício de uma participação autônoma em questões relacionadas à vida familiar, conjugal, ao trabalho, à habilidade de negociação sexual e reprodutiva e, podemos incluir também, ao acesso e utilização de serviços preventivos, como o teste de Papanicolaou. Além disso, os serviços de saúde devem reconhecer e respeitar a bagagem cultural, religiosa e moral das mulheres, propondo um espaço de reflexão e discussão sobre crenças, valores e atitudes das mulheres em relação à saúde e ao seu cuidado, quanto às origens destas crenças, pois muitas dessas, como vimos, advêm de experiências prévias negativas durante as ações de intervenção técnica, bem como quanto as suas conseqüências positivas ou negativas à saúde. Deve-se também trazer para pauta de discussões a violência nas relações de gênero no âmbito doméstico e acolher esse problema como uma demanda legítima à assistência médica, e contribuir para que as próprias mulheres sejam sujeitos ativos no processo de transformação nas relações não eqüitativas entre os gêneros, tanto no âmbito doméstico, quanto no interior das instituições médicas. Os serviços de saúde também poderiam ter um papel ativo rumo a essa transformação, construindo, por exemplo, espaços de diálogo entre homens e mulheres dentro das atividades assistenciais e que estes, por sua vez, possam refletir na geração ou fortalecimento do diálogo no âmbito doméstico. Além disso, deveriam estimular a participação dos homens nos programas em saúde da mulher, trazendo para pauta não somente questões relacionadas às esferas sexual e reprodutiva, mas também questões sobre prevenção à doenças, entre elas, as infecções sexualmente transmissíveis, como a infecção por HPV, fator causal central no desenvolvimento de lesões cervicais neoplásicas.

No intercâmbio entre o plano social e programático, para que os programas de prevenção ao câncer cérvico-uterino obtenham êxito no controle dessa doença mais do que soluções técnicas e econômicas, baseadas na lógica epidemiológica do 
risco e da relação custo-benefício, há a necessidade de se conciliar soluções políticas, não apartadas do compromisso ético, respeitando a autonomia individual das mulheres, dando-lhe um espaço, que lhe é de direito, de participação ativa e autônoma no processo decisório em relação à realização do teste de Papanicolaou ou de outras atividades relacionadas à sua saúde. Nesta direção, torna-se imperativo o compartilhamento das fontes de conhecimento e informação entre as usuárias e profissionais de saúde, para que as mulheres tenham realmente uma participação mais ativa neste processo. Isto significa desmonopolizar ou democratizar 79 o conhecimento médico e epidemiológico e torná-lo acessível à população usuária feminina, como, por exemplo, a informação sobre a importância que a infecção por HPV adquire no processo de carcinogênese cervical e sua transmissão sexual, enfatizando, assim, as estratégias de prevenção primária ao câncer cervical, como o uso de preservativos femininos ou masculinos, e informar também claramente todos os benefícios e potenciais malefícios do teste de Papanicolaou ou de outras técnicas mais invasivas antes de sua aplicação.

Juntos, os três planos, sob o modelo "contextual" proposto, interagem para caracterizar o acesso e a utilização de serviços de saúde em geral e, em particular, dos serviços preventivos, devendo ser compreendidos numa análise que se pretenda totalizadora e não simpatizante de uma visão reducionista dessa questão. Mas, cabe ressaltar que tal modelo não tem a pretensão de se tornar unívoco em sua construção, visto que caracterizar o acesso ao cuidado à saúde em geral é uma tarefa complexa, já que a própria definição de acesso à saúde tem sido objeto de extensos debates na literatura científica na tentativa de conceituá-la e operacionalizá-la e várias são as definições e propostas neste sentido, como a de Puentes-Markides57 e Penchansky e Thomas. 76 Tal modelo antes caracteriza-se como uma proposta aglutinadora e intercambial de diversas contribuições de campos disciplinares como a epidemiologia, as ciências sociais, a economia, entre outros, os quais nos revelam que tomar as características e comportamentos individuais das usuárias como o cerne da questão sobre o acesso a serviços preventivos e considerá-las como apartadas de um contexto social mais amplo é limitada.

Torna-se essencial, pois, que os estudos, na tentativa de caracterizar e explicar o acesso e utilização do teste de Papanicolaou, considerem esse contexto e busquem a inserção de outras esferas discursivas e metodológicas ao desenvolver seus modelos explicativos, a fim de que possam guiar, de forma mais coerente, as intervenções técnicas em saúde pública. Do ponto de vista teórico-metodológico, isto significa "importar" outros olhares à questão, advindos dos mais diversos campos disciplinares, conjugando diferentes métodos de pesquisa, como a aplicação de técnicas de análise quantitativa aliada às abordagens de natureza qualitativa. Ademais, o modelo teórico proposto pode respaldar ou embasar diferentes técnicas de análise estatística dos fatores associados à realização do teste de Papanicolaou, nos quais possam ser contemplados os diferentes níveis ou planos aqui propostos, como os modelos logístico-hierárquicos e os de multi-nível.80 Sob tais perspectivas teóricas e metodológicas, poder-se-á compreender que o êxito no rastreamento do câncer cérvico-uterino dependerá, acima de tudo, do estabelecimento de políticas públicas que visem à redução da vulnerabilidade individual, organizacional/programática e social a que estão potencialmente expostas as mulheres. É fundamental o estabelecimento de intervenções mais humanizadas e eqüitativas, o reconhecimento $d$ as mulheres como sujeitos ativos rumo à conquista de seu bem-estar, o reconhecimento das questões éticas, culturais e político-econômicas que tangenciam as políticas de prevenção e controle do câncer cérvicouterino devendo ser problematizadas nos três planos de análise, aqui, propostos, bem como do compromisso dos agentes responsáveis pelas políticas de saúde em proporcionar aos indivíduos condições sociais e econômicas favoráveis ao exercício pleno de um maior controle sobre sua saúde.

\section{Referências}

1. Parkin DM, Pisani P, Ferlay J. Estimates of the worldwide incidence of 25 major cancers in 1990. Int J Cancer 1999; 80: 827-41.
2. Parkin DM, Whelan SL, Ferlay J, editors. Cancer incidence in five continents. Lyon: IARC; 1997. (IARC Scientific Publication, 143). 
3. Eluf-Neto J, Nascimento CMR. Cervical cancer in Latin America. Semin Oncol 2001; 28: 188-97.

4. SEADE (Fundação Sistema Estadual de Análise de Dados). Produtos SEADE. São Paulo; 2000. Disponível em: <URL: http:IIwww. seade.gov.br\mulher.htm> [2000 Mar 18].

5. São Paulo (Estado). Secretaria de Saúde. Grupo de Assessoria Técnica em Saúde. Coordenação de Planejamento em Saúde. Estratégias para redução da mortalidade por câncer de colo do útero no Estado de São Paulo. São Paulo; 1998.

6. Gustafsson L, Pontén J, Zack M, Adami HO. International incidence rates of invasive cervical cancer after introduction of cytological screening. Cancer Causes Control 1997; 8: 755-63.

7. Day NE. The epidemiological basis for evaluating different screening policies. In: Hakama M, Miller AB, Day NE, editors. Screening for cancer of the uterine cervix. Lyon: IARC ; 1986. (IARC Scientific Publication, 76).

8. Eluf-Neto J, Booth M, Muñoz N, Bosch FX, Meijer CJLM, Walboomers JMM. Human Papillomavirus and invasive cervical cancer in Brazil. Br J Cancer 1994; 69: 114-9.

9. Herrero R, Brinton LA, Reeves WC, Brenes MM, de Britton RC, Gaitan E. Screening for cervical cancer in Latin America: a case-control study. Int J Epidemiol 1992; 21: 1050-6.

10. Soost HJ, Lange HJ, Lehmacher W, Ruffing-Krellmann B. The validation of cervical cytology: sensitivity, specificity and predictive values. Acta Cytol 1991; 35: 8-13.

11. Day NE. Screening for cancer of the cervix. J Epidemiol Community Health 1989; 43: 103-6.

12. WHO (World Health Organization). Cytological screening in the control of cervical cancer: technical guidelines. Genebra; 1988.

13. Linos A, Riza E. Comparisons of cervical cancer programmes in the European Union. Eur J Cancer 2000; 36: 2260-5.

14. Zoorob R, Anderson R, Cefalu C, Sidani M. Cancer screening guidelines. Am Fam Physician 2001; 63: 1101-12.

15. Ministério da Saúde. Centro de Documentação. Assistência integral à saúde da mulher: bases de ação programática. Brasília, DF; 1984.

16. Ministério da Saúde, INCA (Instituto Nacional do Câncer), Coordenadoria de Programas de Controle do CâncerPro-Onco. Viva Mulher: Programa Nacional de Controle do Câncer de Colo Uterino. Rio de Janeiro; 1996.

17. Pinho AA, França-Junior I. Exame de Papanicolaou: por que elas não fazem? Uma revisão da literatura epidemiológica [resumo]. Cienc Saúde Coletiva 2000; 5 Supl: 84.

18. Harlan LC, Bernstein AB, Kessler LG. Cervical cancer screening: who is not screened and why? Am J Public Health 1991; 81: 885-90.

19. Kirkman-Liff B, Kronenfeld JJ. Access to cancer screening services for women. Am J Public Health 1992; 82: 733-5.

20. Bostick RM, Sprafka JM, Virnig BA, Potter JD. Knowledge, attitudes, and personal practices regarding prevention and early detection of cancer. Prev Med 1993; 22: 65-85.

21. Wilcox LS, Mosher WD. Factors associated with obtaining health screening among women of reproductive age.
Public Health Rep 1993; 108: 76-86.

22. Pérez-Stable EJ, Otero-Sabogal R, Sabogal F, McPhee SJ, Hiatt RA. Self-reported use of cancer screening tests among lations and anglos in prepaid health plan. Arch Intern Med 1994; 154: 1073-81.

23. Kottke TE, Trapp MA, Fores MM, Kelly AW, Jung SH, Novotny PJ, Panser LA. Cancer screening behaviors and attitudes of women in southeastern Minnesota. JAMA 1995; 273: 1099-105.

24. Chavez LR, Hubbell FA, Mishra SI, Valdez RB. The influence of fatalism on self-reported use of Papanicoloau smears. Am J Prev Med 1997; 13: 418-24.

25. McPhee SJ, Bird JA, Davis T, Há NT, Jenkins CNH, Le B. Barriers to breast and cervical cancer screening among vietnamese-american women. Am J Prev Med 1997; 13: 205-13.

26. O'Malley AS, Mandelblatt J, Gold K, Cagney KA, Kerner $\mathrm{J}$. Continuity of care and the use of breast and cervical cancer screening services in a multiethnic community. Arch Intern Med 1997; 157: 1462-70.

27. Wismer BA, Moskowitz JM, Chen AM, Kang SH, Novotny TE, Min K, Lew R, Tager IB. Rates and independent correlates of pap smear testing among Korean-American women. Am J Public Health 1998; 88: 656-9.

28. Risendal B, DeZapien J, Fowler B, Papenfuss M, Giuliano A. Pap smear screening among urban Southwestern American Indian women. Prev Med 1999; 29: 510-8.

29. Simões EJ, Newschaffer CJ, Hagdrup N, Ali-Abarghoui F, Tao X, Mack N, Brownson RC. Predictors of compliance with recommended cervical cancer screening schedule: a population-based study. J Community Health 1999; 24: 115-30.

30. Mandelblatt J, Gold K, O'Malley AS, Taylor K, Cagney K, Hopkins JS, Kerner J. Breast and cervix cancer screening among multiethnic women: role of age, health, and source of care. Prev Med 1999; 28: 418-25.

31. Zambrana RE, Breen N, Fox AS, Gutierrez-Mohamed. Use of cancer screening practices by hispanic women: analysis by subgroup. Prev Med 1999; 29: 466-77.

32. Katz S, Hofer TP. Socioeconomic disparities in preventive care persist despite universal coverage. JAMA 1994; 272: $530-4$

33. Camirand J, Potvin L, Béland F. Pap recency: modeling women's characteristics and their patterns of medical care use. Prev Med 1995; 24: 259-69.

34. Ronco G, Segnan N, Ponti A. Who has Pap tests? Variables associated with the use of pap tests in absence of screening programmes. Int J Epidemiol 1991; 20: 349-53.

35. Schwartz M, Savage W, George J, Emohare L. Women's knowledge and experience of cervical screening: a failure of health education and medical organization. Community Med 1989; 11: 279-89.

36. Laczano-Ponce EC, Nájera-Aguilar P, Buiatti E, Alonsode-Ruiz P, Kuri P, Cantoral L. The cervical cancer screening program in Mexico: problems with access and coverage. Cancer Causes Control 1997; 8: 698-704.

37. Cancio JAR, Gallardo HG, Macedo MAS. Factores en la no utilización de detección oportuna del cáncer cervicouterino en medicina familiar. Rev Med IMSS [Mexico] 1997; 35: 227-32.

38. Hernandez-Hernandez DM, Garcia-Elizondo MR, OrnelasBernal L, Hernandez-Aleman F, Gonzalez-Lira G, Martinez-Garcia MC. Factors associated with non-use of 
Pap test. a population survey. Arch Med Res 1998; 29: 263-70

39. Pinotti JA, Faúndes A, Hardy EE, Simões IR, Osis MJD, de Souza TR, Moraes TM. Avaliação da assistência ginecológica no estado de São Paulo. Rev Ginecol Obstet 1990; 1: 7-21.

40. Lopes ER, Rebelo MS, de Abreu E, da Costa Silva VL, Eisemberg ALA, Lavor MF. Comportamento da população brasileira feminina em relação ao câncer cérvicouterino. J Bras Ginecol 1995; 105: 505-16.

41. Nascimento CM, Eluf-Neto J, Rego RA. Cobertura do teste de Papanicolaou no município de São Paulo e características das mulheres que realizaram o teste. Bol Ofic Sanit Panam 1996; 121: 491-99.

42. Karam SM, Horta BL, Gheling CR. Prevenção do carcinoma do colo uterino numa unidade sanitária da UFPel. Rev Bras Cancerol 1996; 42: 87-91.

43. Merighi MAB, Hoga LAK, Praça NS. Detecção precoce do câncer cérvico-uterino numa unidade básica de saúde: uma estratégia de ensino. Mundo Saúde 1997; 21: 300-6.

44. Dias da Costa JS, D' Elia PB, Manzolli P, Moreira MR. Cobertura do exame citopatológico na cidade de Pelotas, Brasil. Rev Panam Salud Publica 1998; 3: 30813.

45. Pinho AA. Fatores associados à realização do teste de $\mathrm{Pa}$ panicolaou entre mulheres em idade reprodutiva no município de São Paulo [dissertação mestrado]. São Paulo: Faculdade de Saúde Pública; 2002.

46. Cockburn J, White VM, Hirst S, Hill D. Barriers to cervical screening in older women. Aust Fam Physician 1992; 21: 973-8.

47. Calle EE, Flanders D, Thun MJ, Martin LM. Demografic predictors of mammography and Pap smear screening in US women. Am J Public Health 1993; 83: 53-60.

48. Lantz PM, Weigers ME, House JS. Education and income differentials in breast and cervical cancer screening. Med Care 1997; 35: 219-36.

49. Peters RK, Bear MB, Thomas D. Barriers to screening for cancer of the cervix. Prev Med 1989; 18: 133-46.

50. Hubbell FA, Chavez LR, Mishra SI, Valdez RB. Beliefs about sexual behavior and other predictors of Papanicolaou smear screening among Latinas and Anglo women. Arch Intern Med 1996; 156: 2353-8.

51. Lee MC. Knowledge, barriers, and motivators related to cervical cancer screening among Korean-American women. Cancer Nurs 2000; 23: 168-75.

52. Seow A, Wong ML, Smith WCS, Lee HP. Beliefs and attitudes as determinants of cervical cancer screening: a community-based study in Singapore. Prev Med 1995; 24: 134-41.

53. Suarez L, Roche RA, Nichols D, Simpson DM. Knowledge, behavior, and fears concerning breast and cervical cancer among older low-income Mexican-American women. Am J Prev Med 1997; 13: 137-42.

54. Laczano-Ponce EC, Castro R, Allen B, Nájera P, Alonsode-Ruiz P, Hérnandez-Avila M. Barriers to early detection of cervical-uterine cancer in Mexico. J Women's Health 1999; 8: 399-108.

55. Becker MH, Rosenstock IM. Selected psychosocial models and correlates of individual health-related behaviors. Med Care 1977; 15 Suppl: 27-46.

56. Castiel LD. A medida do possível... saúde, risco e tecno- biociências. In: Castiel LD. Vivendo entre exposições e agravos: a teoria da relatividade do risco. Rio de Janeiro: Fiocruz; 1999. p. 37-66.

57. Puentes-Markides C. Women and access to health care. Soc Sci Med 1992; 35: 619-26.

58. Yi JK. Factors affecting cervical cancer screening behavior among Cambodian women in Houston, Tex. Fam Community Health 1996; 18: 49-57.

59. Lobell M, Bay C, Rhoads KVL, Keske B. Barriers to cancer screening in Mexican-American women. Mayo Clinic Proc 1998; 73: 301-8.

60. Mann J, Tarantola DJM, Netter TW. A AIDS no mundo. Rio de Janeiro: Relume-Dumará; 1993.

61. Ayres JRCM, França-Junior I, Calazans G. Vulnerabilidade e prevenção em tempos de AIDS. In: Barbosa RM, Parker R., organizadores. Sexualidades pelo avesso: direitos, identidades e poder. Rio de Janeiro: UERJ; 1999. p. $50-72$.

62. Calnan M, Rutter DR. Do health beliefs predict health behaviour? An analysis of breast self-examination. Soc Sci Med 1986; 22: 673-8.

63. Lupton D. Risk as moral danger: the social and political functions of risk discourse in public health. Int J Health Serv 1993; 23: 425-35.

64. Bush J. It's just part of being a woman: cervical screening, the body and feminity. Soc Sci Med 2000; 50: 429-44.

65. Hankins C, Coutlée F, Lapointe N, Simord P, Tran T, Samson T. Prevalence of risk factors associated with human papillomavirus infection in women living with HIV. CMAJ 1999; 160: 185-91.

66. Ho GYF, Burk RD, Fleming I, Klein RS. Risk of genital human papillomavirus infection in women with human immunodeficiency virus-induced immunosuppression. Int J Cancer 1994; 56: 788-92.

67. Barbosa RM. Negociação sexual ou sexo negociado? Poder, gênero e sexualidade em tempos de AIDS. In: Barbosa RM, Parker R., organizadores. Sexualidades pelo avesso: direitos, identidades e poder. Rio de Janeiro: UERJ; 1999. p. 50-72.

68. Heise L, Ellsberg M, Gottemoeller M. Ending violence against women. Popul Reports 1999; (11), Série L

69. Tollestrup K, Sklar D, Frost FJ, Olson L, Weybright J, Sandvig J, Larson M. Health indicators and intimate partner violence among women who are members of a managed care organization. Prev Med 1999; 29: 431-40.

70. Eardley A, Elkind AK, Spencer B, Hobbs P, Pendleton LL, Haran D. Attendance for cervical screening-whose problem? Soc Sci Med 1985; 20: 955-62.

71. Soost HJ, Lange HJ, Lehmacher W, Ruffing-Krellmann B. The validation of cervical cytology: sensitivity, specificity and predictive values. Acta Cytol 1991; 35: 8-13.

72. DiBonito L. Cervical cytopathology: an evaluation of its accuracy based on cytohistologic comparison. Cancer 1993; 72: 3002-6.

73. Chamberlain J. Reasons that some screening programmes fail to control cervical cancer. In: Hakama M, Miller $\mathrm{AB}$, Day NE, editors. Screening for cancer of the uterine cervix. Lyon: IARC; 1986. (IARC Scientific Publication, 76).

74. Wilson RM. Screening for breast and cervical cancer as a common cause for litigation [editorial). BMJ 2000; 320: 1352-3.

75. Austin RM, Mclendon WW. The Papanicolaou smear: 
medicine's most successful cancer screening procedure is threatened [editorial]. JAMA 1997; 277: 754-5.

76. Penchansky R, Thomas W. The concept of access. Med Care 1981; 19: 127-40.

77. D'Oliveira AFPL, Senna DM. Saúde da mulher. In: Schraiber LB, Nemes BMI, Mendes-Gonçalves RB, organizadores. Saúde do adulto: programas e ações na unidade básica. São Paulo: Hucitec; 1996. p. 86-108.

78. Osis MJMD. PAISM: um marco na abordagem da saúde reprodutiva no Brasil. Cad Saúde Pública 1998; 14 [Supl 1]: 25-32.
79. Schraiber LB, D' Oliveira AFPL. Violência contra mulheres: interfaces com a saúde. Interface Comun Saúde Educ 1999; 3: 11-26.

80. Goldstein H. Multilevel statistical models. 2. ed. Londres: Edward Arnold; 1995.

Recebido em 10 de setembro de 2002

Versão final reapresentada em 16 de novembro de 2002

Aprovado em 6 de dezembro de 2002 